Fortschritt-Berichte VDI

VDI

Reihe 12

Verkehrstechnik/ Fahrzeugtechnik

Nr. 804
Michael Aeberhard, M.Sc., München

Object-Level Fusion for Surround Environment Perception in Automated Driving Applications 
https://doi.org/10.51202/9783186804129-1

Generiert durch IP '172.22.53.54', am 26.04.2023, 14:43:27

Das Erstellen und Weitergeben von Kopien dieses PDFs ist nicht zulässig. 


\title{
Object-Level Fusion for Surround Environment Perception in Automated Driving Applications
}

\author{
DISSERTATION \\ submitted in partial fulfillment \\ of the requirements for the degree \\ Doktor Ingenieur \\ (Doctor of Engineering)
}

in the

\section{Faculty of Electrical Engineering and Information Technology} at Technische Universität Dortmund

\section{by}

M.Sc. Michael Aeberhard

Lausanne, Switzerland

Date of approval: May 31st, 2017

First examiner: Univ.-Prof. Dr.-Ing. Prof. h.c. Dr. h.c. Torsten Bertram

Second examiner: Univ.-Prof. Dr.-Ing. Hans-Joachim Wünsche 
https://doi.org/10.51202/9783186804129-1

Generiert durch IP '172.22.53.54', am 26.04.2023, 14:43:27

Das Erstellen und Weitergeben von Kopien dieses PDFs ist nicht zulässig. 


\section{Fortschritt-Berichte VDI}

Reihe 12

Verkehrstechnik/

Fahrzeugtechnik

Nr. 804
Michael Aeberhard, M.Sc., München

Object-Level Fusion for Surround Environment Perception in Automated Driving Applications

vDI verlag 
Aeberhard, Michael

\section{Object-Level Fusion for Surround Environment Perception in Automated Driving Applications}

Fortschr-Ber. VDI Reihe 12 Nr. 804. Düsseldorf: VDI Verlag 2017. 214 Seiten, 79 Bilder, 21 Tabellen.

ISBN 978-3-18-380412-2, ISSN 0178-9449,

$€ 76,00 / \mathrm{VDI}$-Mitgliederpreis $€ 68,50$.

Keywords: Sensor Fusion - Perception - Autonomous Driving - Driver Assistance - Track-toTrack Fusion - Tracking - Object Detection

Driver assistance systems have increasingly relied on more sensors for new functions. As advanced driver assistance system continue to improve towards automated driving, new methods are required for processing the data in an efficient and economical manner from the sensors for such complex systems. In this thesis, an environment model approach for the detection of dynamic objects is presented in order to realize an effective method for sensor data fusion. A scalable high-level fusion architecture is developed for fusing object data from several sensors in a single system. The developed high-level sensor data fusion architecture and its algorithms are evaluated using a prototype vehicle equipped with 12 sensors for surround environment perception. The work presented in this thesis has been extensively used in several research projects as the dynamic object detection platform for automated driving applications on highways in real traffic.

\section{Bibliographische Information der Deutschen Bibliothek}

Die Deutsche Bibliothek verzeichnet diese Publikation in der Deutschen Nationalbibliographie; detaillierte bibliographische Daten sind im Internet unter http://dnb.ddb.de abrufbar.

\section{Bibliographic information published by the Deutsche Bibliothek}

(German National Library)

The Deutsche Bibliothek lists this publication in the Deutsche Nationalbibliographie (German National Bibliography); detailed bibliographic data is available via Internet at http://dnb.ddb.de.

Alle Rechte, auch das des auszugsweisen Nachdruckes, der auszugsweisen oder vollständigen Wiedergabe (Fotokopie, Mikrokopie), der Speicherung in Datenverarbeitungsanlagen, im Internet und das der Übersetzung, vorbehalten. 


\section{Acknowledgments}

This thesis and the corresponding research was completed with the Technische Universität Dortmund in cooperation with the BMW Group Research and Technology in Munich, Germany.

First and foremost I would like to thank Univ.-Prof. Dr.-Ing. Prof. h.c. Dr. h.c. Torsten Bertram for advising this thesis and guiding me through the process of undergoing the research and writing the thesis. I am grateful for his many insights, advice and suggestions while completing the thesis. Additionally I would like to thank Univ.-Prof. Dr.-Ing. HansJoachim Wünsche of the Universität der Bundeswehr for taking over the role as second examiner.

I would like to thank Dr.-Ing. Dirk Wisselmann, Dr.-Ing. Helmut Spannheimer and Dr.-Ing. Nico Kaempchen for giving me the chance to write my thesis in cooperation with the BMW Group and giving me the opportunity to begin my career in the exciting and fulfilling field of autonomous driving. My sincere thanks go in particular to Dr.-Ing. Nico Kaempchen, who served as my technical adviser at BMW during the time as a doctoral student. Without his guidance, insights, experience and encouragement during the scores of meetings, discussions and test drives, this thesis would not have been possible.

Additionally I would like to thank my fellow colleagues at BMW with whom many exciting projects were completed: Michael Ardelt, Mohammad Bahram, Martin Friedl, Thomas Hofmann, Florian Homm, Pei-Shih Huang, Werner Huber, Horst Klöden, Thomas Kühbeck, Yves Pilat, Andreas Rauch, Sebastian Rauch, Bernhard Seidl, Georg Tanzmeister, Julian Thomas, Peter Waldmann and Moritz Werling. I am also grateful for the excellent contributions of all my bachelor/master/diploma thesis students: Christopher Bayer, Sebastian Dingler, Sascha Paul, Marcin Rabiega, Stefan Schlichthärle and Jinquan Zheng.

My experience with BMW began at the manufacturing plant in Spartanburg, South Carolina as an intern while studying at the Georgia Institute of Technology in Atlanta, GA. For this opportunity, I am thankful to Russell Levesque, my adviser during the internship. I am also thankful to Prof. Dr. Markus Krug, now a Professor at the University of Applied Sciences Munich, for giving me the opportunity to do an international internship at BMW Motorsport in Munich, Germany, which sparked my interest in working in Germany. I would also like to thank Dr.-Ing. Marc Muntzinger for giving me the opportunity to complete my master thesis at Daimler AG in Ulm, Germany, where I first gained experience in the field of driver assistance systems.

I am very grateful and forever indebted to my parents, Brigitte Aeberhard and Peter Aeberhard, for their continued support throughout my life and always believing in me during my endeavors. Last but not least I would like to thank my wife Rabea for her love, support and encouragement during the final stretches of completing this thesis. 
66 For once you have tasted flight you will walk the earth with your eyes turned skywards, for there you have been and there you will long to return.

Leonardo da Vinci 


\section{Contents}

$\begin{array}{lll}\text { Abbreviations } & \text { VIII }\end{array}$

List of Symbols $\quad X$

$\begin{array}{ll}\text { Abstract } & \text { XV }\end{array}$

1 Introduction 1

1.1 Motivation . . . . . . . . . . . . . . . . . . . . 2

1.2 Automation in Driver Assistance and Safety Systems . . . . . . . . . . 3

1.2.1 Level 0 - No Automation . . . . . . . . . . . . . . . . 6

1.2.2 Level 1 - Driver Assistance . . . . . . . . . . . . . . . . . 7

1.2.3 Level 2 - Partial Automation . . . . . . . . . . . . . . . . . 8

1.2.4 Level 3 - Conditional Automation . . . . . . . . . . . . . . . 9

1.2.5 Levels 4 and 5 - Towards Fully Automated Driving . . . . . . . . 15

1.3 Problem of Object Detection . . . . . . . . . . . . . . . . . . . . . . . . . . . . . . . 21

1.4 Contribution and Outline of the Thesis . . . . . . . . . . . . . . 23

2 Sensor Data Fusion Architectures $\quad 27$

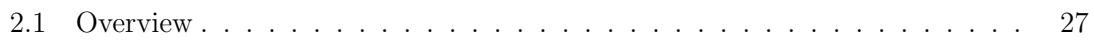

2.1.1 Low-Level . . . . . . . . . . . . . . . . . . . . . . . . . . . . . . . . . . . . . 28

2.1 .2 High-Level . . . . . . . . . . . . . . . . . . . . . . 31

2.1 .3 Hybrid . . . . . . . . . . . . . . . . . . . . . 32

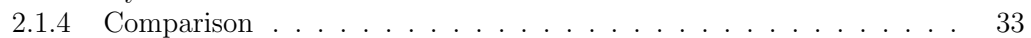

2.2 Proposed Modular Sensor Data Fusion Architecture . . . . . . . . . . . . . . . . . 38

2.2.1 Object Model . . . . . . . . . . . . . . . . . . . . . . . . . . 39

2.2 Sensor-Level . . . . . . . . . . . . . . . . . . . . . . 41

2.2 .3 Fusion-Level . . . . . . . . . . . . . . . . . . . . . . . . 42

2.2 .4 Application-Level . . . . . . . . . . . . . . . . . . 43

3 Fusion Strategy and Object Association 46

3.1 Data Alignment . . . . . . . . . . . . . . . . . . . . 46

3.1 .1 Spatial . . . . . . . . . . . . . . . . . . 46

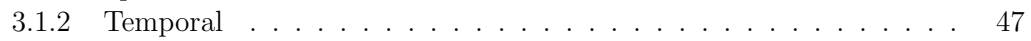

3.2 Fusion Strategy . . . . . . . . . . . . . . . . . . . . . . . . . . . . . . . . . . . . . . . . . . . 48

3.2.1 Sensor-to-Sensor . . . . . . . . . . . . . . . . . . 48

3.2 .2 Sensor-to-Global . . . . . . . . . . . . . . . . . . . . . . . . . . . . . . . 49

3.3 Association . . . . . . . . . . . . . . . . . . . . . . 50

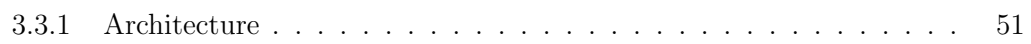

3.3 .2 Feature Selection . . . . . . . . . . . . . . . . . . . . . . 52

3.3 .3 State Vector . . . . . . . . . . . . . . . . . . . . 56

3.3 .4 Geometrical . . . . . . . . . . . . . . . . 58 
3.3.5 Association Validation . . . . . . . . . . . . . . . . . . 60

3.3.6 Multi-Object Association . . . . . . . . . . . . . . . . . 60

4 State and Covariance $\quad 64$

4.1 Sensor-Level Processing with Tracking Algorithms . . . . . . . . . . . . . . 64

4.1.1 Feature Extraction . . . . . . . . . . . . . . . . . . 65

4.1 .2 Data Association . . . . . . . . . . . . . . . 66

4.1 .3 Filtering . . . . . . . . . . . . . . . . . . 66

4.1 .4 Track Management . . . . . . . . . . . . . . . . . . . . 68

4.1.5 Kinematic Models . . . . . . . . . . . . . . . . . . . 68

4.2 Correlation and Sequence of Sensor Data . . . . . . . . . . . . . . . . . . . . . . . . . . . . . . . . 69

4.2 .1 Process Noise . . . . . . . . . . . . . . . . . . . . . . . . . . . . . . . 70

4.2 .2 Common Information History . . . . . . . . . . . . . . . . . . . 71

4.2 .3 Out-of-Sequence Data . . . . . . . . . . . . . . . . 72

4.3 Track-to-Track Fusion with the Common State . . . . . . . . . . . . . . . . 73

4.3 .1 Adapted Kalman Filter . . . . . . . . . . . . . . . . . . . . . . 74

4.3 .2 Covariance Intersection . . . . . . . . . . . . . . . . . . . 76

4.3 .3 Information Matrix Fusion . . . . . . . . . . . . . . . . . . . . 77

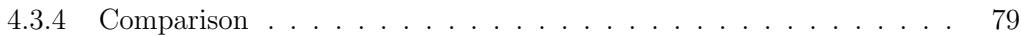

4.4 Geometrical Fusion using the Object Model . . . . . . . . . . . . . . . . . . 86

4.4 Dimension Estimation . . . . . . . . . . . . . . . . 87

4.4 .2 Extraction of Fused Coordinates . . . . . . . . . . . . . . . . 90

5 Existence Probability $\quad 93$

5.1 Sensor-Level Processing . . . . . . . . . . . . . . . . . . . . . . . . 93

5.1 .1 Existence Prediction . . . . . . . . . . . . . . . . . . . . 94

5.1 .2 Existence Update . . . . . . . . . . . . . . . . . . . . . . . . . . . . 95

5.1.3 Generalized Bayes Extension . . . . . . . . . . . . . . . . . . . 97

5.1 .4 Modeling the Parameters . . . . . . . . . . . . . . . . . . . . . . 98

5.1 .5 Object Management . . . . . . . . . . . . . . . . . . . . . . . 104

5.2 Fusion . . . . . . . . . . . . . . . . . . . . . 105

5.2.1 Architecture . . . . . . . . . . . . . . . . . 105

5.2.2 Modeling with Dempster-Shafer Evidence Theory . . . . . . . . . . 105

5.2.3 Extension for Occlusion Modeling . . . . . . . . . . . . . . . . . 110

5.2.4 Modeling the Trust Probability . . . . . . . . . . . . . . . . . . . . 114

6 Classification $\quad \mathbf{1 1 6}$

6.1 Sensor-Level Processing . . . . . . . . . . . . . . . . . . . 117

6.1.1 Measurement Classification . . . . . . . . . . . . . . . . 117

6.1 .2 Temporal Filtering . . . . . . . . . . . . . . . . . . . . . 124



6.2.1 Modeling with the Dempster-Shafer Evidence Theory . . . . . . . . 125

6.2.2 Modeling the Trust Probability . . . . . . . . . . . . . . . . 130

7 Evaluation 133

7.1 Test Vehicle and Sensor Configuration . . . . . . . . . . . . . . 133 
7.2 Overtaking Maneuver with Ground Truth . . . . . . . . . . . . . . 135

7.2.1 Ground Truth Calculation . . . . . . . . . . . . . . . . 136

7.2 .2 State Estimation . . . . . . . . . . . . . . . . . . 136

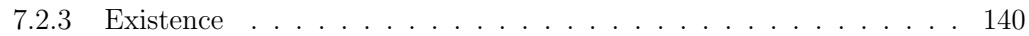

7.2 .4 Classification . . . . . . . . . . . . . . . . . . . 140

7.3 Performance in Real Traffic Scenarios . . . . . . . . . . . . . . . . . . . . . 141

7.3 .1 Detection Rate . . . . . . . . . . . . . . . . . . . . . . 144

7.3.2 Classification Performance . . . . . . . . . . . . . . . . 146

7.3.3 Integration in Automated Driving and ADAS Projects . . . . . . . 149

8 Conclusion and Discussion 152

A Synchronous Track-to-Track Fusion Algorithms 155

A.1 Simple Weighted Fusion . . . . . . . . . . . . . . . . . . . . 155

A.2 Use of Cross-Covariance . . . . . . . . . . . . . . . . . . . . . . 157

A.3 Covariance Intersection . . . . . . . . . . . . . . . . . . . . . . . 158

A.4 Comparison . . . . . . . . . . . . . . . . . . . 159

B Information Matrix Fusion Derivation $\quad 162$

C Determining the Trust Probability 164

C.1 Existence . . . . . . . . . . . . . . . . . . . . . . 164

C.2 Classification . . . . . . . . . . . . . . . . . . 165

D Evaluation Scenario Descriptions 167

D.1 Training Data . . . . . . . . . . . . . . . . . . . . . 167

D.2 Evaluation Data. . . . . . . . . . . . . . . . . . . . . 167

D.2.1 Test Track . . . . . . . . . . . . . . . . . . . . 167

D.2.2 Real Traffic . . . . . . . . . . . . . . . . . . . . . 168

$\begin{array}{ll}\text { References } & 172\end{array}$ 


\section{Abbreviations}

ABS Anti-Lock Brakes.

ACC Active Cruise Control.

ADAS Advanced Driver Assistance Systems.

ALA Active Lane Assist.

ANIS Average Normalized Innovation Squared.

AUC Area Under the Curve.

BASt Bundesanstalt für Straßenwesen.

BBA Basic Belief Assignment.

BSD Blind Spot Detection.

CAN Controller Area Network.

DARPA Defense Advanced Research Projects Agency.

DSC Dynamic Stability Control.

DST Dempster-Shafer Evidence Theory.

EBA Emergency Brake Assist.

ESP Electronic Stability Program.

FCW Forward Collision Warning.

FISST Finite Set Statistics.

GDA Gaussian Discriminant Analysis.

GPS Global Positioning System.

IMM Interacting Multiple Model.

IPDA Integrated Probabilistic Data Association.

JIPDA Joint Integrated Probabilistic Data Association.

JPDA Joint Probabilistic Data Association.

LDW Lane Departure Warning. 
LKA Lane Keeping Assist.

MHT Multiple Hypothesis Tracking.

NEES Normalized Estimation Error Squared.

NHTSA National Highway Traffic Safety Administration.

NIS Normalized Innovation Squared.

PDA Probabilistic Data Association.

PHD Probability Hypothesis Density.

RMSE Root Mean Squared Error.

ROC Receiver Operating Characteristic.

SAE Society of Automotive Engineers.

SVM Support Vector Machines.

TJA Traffic Jam Assist.

V2V Vehicle-to-Vehicle Communication.

VRU Vulnerable Road User. 


\section{List of Symbols}

\section{General Notation}

$a$

a

A

$\mathbf{A}^{\prime}$

$\mathbf{A}^{-1}$

(.)

(.)

(.)

(.) $(k)$

$(.)_{k}$

$().(k \mid k)$

$(.)_{k \mid k}$

(.) $(k \mid k-i)$

$(.)_{k \mid k-i}$

(.) $(k, k-i)$

(.) $(t)$

(.) $(t-\tau)$

$\{()\}.(t)$

$\{(.)\}_{a}^{b}$

$\operatorname{Bel}(()$.

$\operatorname{BetP}(()$.
Scalar

Vector

Matrix

Transpose of matrix A

Inverse of matrix A

Estimate of the true value of (.)

Error between the estimate $($.$) and the true value (.)$

Complement of (.)

Value of (.) at the discrete time step $k$

Value of (.) at the discrete time step $k$

Value of (.) at the discrete time step $k$ conditioned on information from the current time step $k$

Value of (.) at the discrete time step $k$ conditioned on information from the current time step $k$

Value of (.) at the discrete time step $k$ conditioned on information from a previous discrete time step $k-i$

Value of (.) at the discrete time step $k$ conditioned on information from a previous discrete time step $k-i$

Transition from $k-i$ to $k$ with (.)

Value of (.) at the continuous time $t$

Value of (.) at a previous continuous time $t-\tau$

Set of (.) at the continuous time $t$

Complete set of (.) from time $a$ up to time $b$

Belief function

Pignistic transformation of (.) 
E $[()$.$] \quad Expected value of ($.

$F_{(.)}^{-1} \quad$ Inverse cumulative distribution function for the (.) distribution

$m(()$.$) \quad Dempster-Shafer evidence theory basic belief assignment$

$\mathrm{Pl}(()$.$) \quad Plausibility function$

\section{Latin Letters}

$\mathcal{C}$

$\mathcal{D}$

$\mathcal{E}$

$\mathcal{G}$

$\mathcal{I}$

$\mathcal{I}^{*}$

$\mathcal{L}$

$\mathcal{M}$

$\mathcal{O}$

$\mathcal{R}$

$\mathcal{S}$

$\mathcal{T}$

$\mathcal{U}$

$\mathcal{X}$

c

d

$\mathbf{d}_{\sigma^{2}}$

f

m

p

u

w

$\mathrm{x}$

$\mathbf{x}_{\mathrm{a}}$
Set of classification hypothesis

Generic representation for some data

Environment model

Generic representation for spatial-based, or grid-based, objects/obstacles

Generic representation for information

Generic representation for a-priori information

Log odds ratio

Generic representation for digital map information

Object list

Generic representation for road infrastructure information

Generic representation for data perceived from a sensor

Training set

Generic representation for control information from the host vehicle platform

Generic representation for host vehicle localization and pose

Classification vector of an object

Dimension vector of an object

Dimension uncertainty vector of an object

Feature vector of an object

1-dimensional grid map for geometrical dimension estimation

Position vector in a Cartesian coordinate system

Host system control vector

Normal vector to a decision boundary

State vector of an object

State vector subset of $\mathbf{x}$ of an object used for association 
y

Z

A

B

C

F

H

I

K

$\mathrm{S}$

P

$\mathbf{P}^{a b}$

$\mathbf{P}_{a b}$

Q

R

W

$a$

$a_{i, j}$

$w$

$b$

$d$

$d^{2}$

$g$

$l$

m

$n_{\mathbf{a}}$

$p$

$r$

$v$

$x$

$y$

Attribute vector for classification

Measurement vector

Object association matrix

Control transformation matrix

Association cost matrix in the auction algorithm

State transition matrix

State-space transformation matrix

Identity matrix

Kalman gain

Innovation covariance matrix

Covariance matrix of a state estimate $\hat{\mathbf{x}}$

Cross-covariance between the state estimates $\hat{\mathbf{x}}^{a}$ and $\hat{\mathbf{x}}^{b}$

Cross-covariance matrices with retrodicted states

Process noise covariance matrix

Covariance matrix of a measurement $\mathbf{z}$

Kalman gain for an out-of-sequence measurement

Acceleration of an object

The element of the association matrix $\mathbf{A}$ in the $i$ th row and $j$ th column

Width of an object

Bias parameter

Geometrical dimension of an object

Mahalanobis distance

Boolean result from geometrical association

Length of an object

Single cell of the 1-dimensional map $\mathbf{m}$

Number of elements, or dimension, of vector a

Bid price for assignment in the auction algorithm

Range in a polar coordinate system

Velocity of an object

Position of an object on the $x$-axis in a Cartesian coordinate system

Position of an object on the $y$-axis in a Cartesian coordinate system 
$C_{i} \quad$ Object class $i$, where $i$ corresponds to the $i$ th element of $\mathbf{c}$ or $\mathcal{C}$

$D^{2} \quad$ Extended Mahalanobis distance

G Gating threshold during object association

H Object association hypothesis

$O_{i} \quad$ The $i$ th object in an object list $\mathcal{O}$

$Z^{i} \quad$ List of measurements from sensor $i$

\section{Greek Letters}

$\gamma$

$\delta$

$\Delta($.

$\epsilon$

$\eta$

$\theta$

$\Theta$

$\lambda$

$\mu$

$\rho$

$\sigma$

$\sigma^{2}$

$\phi$

$\psi$

$\dot{\psi}$

$\omega$

Dempster-Shafer evidence theory prediction weight

Offset/translation of a sensor's placement on the vehicle

Difference of (.) between two values

Normalized Estimation Error Squared

Normalization factor

Orientation of a sensor's mounting position on the vehicle

Dempster-Shafer evidence theory frame of discernment

Rate parameter of a Poisson process

Mean

Correlation weighting factor

Standard deviation

Variance

Angle in a 2-dimensional polar coordinate system

Orientation angle of an object

Orientation velocity of an object

Covariance intersection weighting factor

\section{Subscripts and Superscripts}

$(.)^{S_{i}}$

$(.)^{\mathrm{G}}$

$(.)^{\text {obj }}$

(.) $)^{\text {sensor }}$

$(.)^{\mathrm{veh}}$
(.) originates from sensor $\mathrm{S}_{i}$

(.) results from a global fusion algorithm

(.) is in the object coordinate system

(.) is in the sensor coordinate system

(.) is in the host vehicle coordinate system 
$(.)_{\mathrm{f}} \quad$ Value of $($.$) corresponds to the feature \mathrm{f}$

$(.)_{x} \quad$ Scalar corresponding to the $x$ component of $($.$) in a Cartesian coordinate$ system

$(.)_{y} \quad$ Scalar corresponding to the $y$ component of (.) in a Cartesian coordinate system

(.) $\mathrm{a} \rightarrow \mathrm{b} \quad$ Transformation from $\mathrm{a}$ to $\mathrm{b}$

\section{Probabilities}

$p(a) \quad$ Continuous probability density function of the random variable $a$

$p(a \mid b) \quad$ Continuous conditional probability density of the random variable $a$ conditioned on $b$

$p(\exists \mathbf{x}) \quad$ Existence probability of an object

$p(\nexists \mathbf{x}) \quad$ Non-existence probability of an object

$p_{\mathrm{b}} \quad$ Birth probability

$p_{\mathrm{c}} \quad$ Clutter probability

$p_{\mathrm{d}} \quad$ Detection probability

$p_{\mathrm{p}} \quad$ Persistence probability

$p_{\text {trust }} \quad$ Trust probability

$P_{(.)} \quad$ Scalar probability value 


\section{Abstract}

Driver assistance systems have increasingly relied on more sensors for new functions. As advanced driver assistance system continue to improve towards automated driving, new methods are required for processing the data in an efficient and economical manner from the sensors for such complex systems. The detection of dynamic objects is one of the most important aspects required by advanced driver assistance systems and automated driving. In this thesis, an environment model approach for the detection of dynamic objects is presented in order to realize an effective method for sensor data fusion. A scalable high-level fusion architecture is developed for fusing object data from several sensors in a single system, where processing occurs in three levels: sensor, fusion and application. A complete and consistent object model which includes the object's dynamic state, existence probability and classification is defined as a sensor-independent and generic interface for sensor data fusion across all three processing levels. Novel algorithms are developed for object data association and fusion at the fusion-level of the architecture. An asynchronous sensor-to-global fusion strategy is applied in order to process sensor data immediately within the high-level fusion architecture, giving driver assistance systems the most up-todate information about the vehicle's environment. Track-to-track fusion algorithms are uniquely applied for dynamic state fusion, where the information matrix fusion algorithm produces results comparable to a low-level central Kalman filter approach. The existence probability of an object is fused using a novel approach based on the Dempster-Shafer evidence theory, where the individual sensor's existence estimation performance is considered during the fusion process. A similar novel approach with the Dempster-Shafer evidence theory is also applied to the fusion of an object's classification. The developed high-level sensor data fusion architecture and its algorithms are evaluated using a prototype vehicle equipped with 12 sensors for surround environment perception. A thorough evaluation of the complete object model is performed on a closed test track using vehicles equipped with hardware for generating an accurate ground truth. Existence and classification performance is evaluated using labeled data sets from real traffic scenarios. The evaluation demonstrates the accuracy and effectiveness of the proposed sensor data fusion approach. The work presented in this thesis has additionally been extensively used in several research projects as the dynamic object detection platform for automated driving applications on highways in real traffic. 
https://doi.org/10.51202/9783186804129-1

Generiert durch IP '172.22.53.54', am 26.04.2023, 14:43:27

Das Erstellen und Weitergeben von Kopien dieses PDFs ist nicht zulässig. 